\title{
Estrategias mediadas por TIC para el desarrollo de enfoque de aprendizaje profundo en estudiantes universitarios
}

\section{Strategies mediated by information and communication technologies, ICT, for the development of a deep learning approach among college students}

\author{
Nohemí Carrascal Torres' \\ Gustavo Alvarino Bettín ${ }^{2}$ \\ Estela Díaz Buitrago ${ }^{3}$
}

Resumen

Este estudio tiene como objetivo validar estrategias mediadas por Tecnologías de la Información y la Comunicación, TIC, para mejorar el desempeño académico en estudiantes del curso Física I, del segundo semestre del Programa de Ingeniería Industrial de la Universidad de Córdoba. Con diseño cuasiexperimental, de preprueba-posprueba y grupo control, se intervino una población de ciento diez estudiantes en el segundo periodo académico de 2007, integrada por cincuenta y cuatro en el grupo experimental y cincuenta y seis en el grupo control; se apoya en la teoría socio-constructiva, en los postulados de Biggs sobre calidad del aprendizaje y se operacionaliza en el Modelo 3P de enseñanza-aprendizaje, midiendo enfoques de aprendizaje, contextos de enseñanza y niveles de comprensión, a partir de la Taxonomía Solo (Biggs y Collins, 1982).

\section{Palabras clave}

Calidad del aprendizaje, enfoques de aprendizaje, niveles de comprensión, estrategias mediadas con TIC; Taxonomía Solo.

Abstract

This study has as main objective to validate the use of ICT to improve second semester Industrial Engineering students' academic performance in a course of Physics I at University of Cordoba, Montería, Colombia. A quasiexperimental research design was followed, using a pre-test, posttest and a control group, with a population of 110 students, 54 in the experimental group and 56 in the control group during the second semester of 2007. This study was theoretical supported by the Socio Constructive Theory, the postulates of Biggs about quality of learning. It works in the teaching and learning Model 3P, measuring approaches of learning, educational contexts and understanding levels from the Solo Taxonomy.

\section{Keywords}

Learning quality, learning approaches, understanding levels, half-full strategies with ict, Solo Taxonomy.

Artículo recibido el 5 de diciembre de 2008 y aprobado el 28 de abril de 2009.

1 Docente titular Departamento de Psicopedagogía de la Universidad de Córdoba. Psicóloga Universidad de Antioquia. Doctora en Educación.

2 Docente asociado al Departamento de Física de la Universidad de Córdoba. Licenciado en Matemáticas Universidad de Córdoba. Especialista en Ciencias Físicas Universidad Nacional de Colombia. Magíster en Ciencias Físicas Universidad Nacional de Colombia. Mg. en Educación sue-CaribeCorreo electrónico: alvarinog@gmail.com

3 Docente ied Camilo Torres. Licenciado en Informática Educativa de la Universidad de Córdoba. Mg. en Educación sue-Caribe. 
Lo que hace el estudiante es, en realidad,

más importante para determinar lo que aprende que lo que hace el profesor.

Thomas J. Shuell

\section{Introducción}

Actualmente, los esfuerzos en el ámbito educativo apuntan hacia la formación de ciudadanos justos, integrales, competentes, emprendedores e innovadores, con recursos cognitivos, cognoscitivos $\mathrm{y}$ actitudinales que les permitan vivir, convivir, adaptarse y contribuir al desarrollo de su entorno, lo cual se resume en lo que se denomina aprendizaje para la vida. A partir de los años ochenta, se ha gestado entre maestros e investigadores el interés por hacer efectivo, eficaz y productivo el proceso de enseñanza-aprendizaje, que ha llevado a interrogar sobre la manera en que el alumno aprende, cómo se desarrollan los procesos de enseñanza y aprendizaje, para, a partir de ahí, tomar decisiones que favorezcan el logro de un aprendizaje de calidad, en el cual el alumno sea el centro del proceso y el docente sea quien diseñe las estrategias para que éste aprenda. De acuerdo con esta perspectiva, surgen líneas de investigación, como la fenomenográfica y la metacognitiva, que, desde las perspectivas metodológicas cualitativa y cuantitativa, abordan los contextos de enseñanza y aprendizaje y las estrategias que median en su interacción.

Enmarcado en el modelo Student Aproaches to Learning, sal, cuyo objetivo es abordar la calidad del aprendizaje desde los desempeños de los estudiantes (Biggs, 1978, Biggs y Collins, 1982), se desarrolla este proceso, que permite intervenir los contextos de enseñanza y aprendizaje, con aplicación y validación de estrategias mediadas con tic, de tal manera que, a partir de la construcción de un prototipo basado en las directrices planteadas en la estrategia, se pueda promover el desarrollo de un aprendizaje de calidad y para toda la vida.

Las TIC desempeñan un papel importante en el desarrollo de procesos de aprendizaje por facilidad de uso y versatilidad; permiten superar dificultades espacio-temporales; su variedad de recursos constituye el soporte apropiado para una comprensión más profunda de los fenómenos y una labor docente personalizada; permiten el diseño de espacios que favorecen el aprendizaje significativo y mejoran la motivación de los estudiantes.

El diseño de este modelo se justifica en la posibilidad de los estudiantes de lograr el desarrollo eficaz del aprendizaje, mediante el seguimiento y valoración de su trabajo, brindándoles las herramientas que facilitan el empleo de estrategias de aprendizaje, el desarrollo de capacidades, habilidades, actitudes y valores que posibiliten la formación en competencias, así como la aplicación de las TIC, para gestionar el conocimiento. En cuanto al docente, los retos se dirigen a abandonar los métodos tradicionales, a aplicar estrategias pedagógicas que desarrollen en los estudiantes la capacidad de aprender a aprender, adaptarse a los cambios sociales, productivos, económicos y tecnológicos, y a transformar la realidad en la que están inmersos. Es decir, el docente deberá gestionar y desarrollar un nuevo quehacer pedagógico.

La viabilidad del trabajo se aborda desde la óptica pedagógica, pues resulta beneficioso en el contexto de la Universidad de Córdoba, donde no se han desarrollado propuestas que integren las TIC a los procesos de enseñanza-aprendizaje en el sistema de créditos, lo cual conduce a un cambio en la manera en que se han venido desarrollando estos procesos, así como la superación de las limitaciones para el desarrollo de aprendizajes desde un nuevo enfoque, que demanda habilidades y actitudes específicas para la gestión del conocimiento. Además, se ajusta a los lineamientos de los planes y estrategias planteados por el Ministerio de Educación Nacional en su Plan Visión Colombia 2019.

La investigación se produce en el contexto de la Maestría en Educación suE-Caribe y constituye un aporte a la investigación educativa en la región, en cuanto contribuye al mejoramiento de la calidad de la educación, a la construcción de un modelo que, desde lo institucional, incide en lo regional y a la transformación de los procesos de enseñanza y aprendizaje en el ámbito de la educación superior, 
dado que, a nivel regional, las experiencias de estrategias mediadas por TIC articuladas a los contextos de enseñanza y aprendizaje son escasas y aisladas.

El trabajo se propuso alcanzar los siguientes objetivos:

Objetivo general:

- Evaluar estrategias mediadas con TIC, para promover un mejor desempeño académico en estudiantes del curso de Física I del programa de Ingeniería Industrial de la Universidad de Córdoba.

Objetivos específicos:

- Identificar las estrategias de aprendizaje y los contextos de enseñanza de los estudiantes de Física I del Programa, para determinar las condiciones internas y externas al iniciar el proceso.

- Modelar técnica y pedagógicamente el ambiente de aprendizaje, basado en aplicación de estrategias mediadas con TIC para mejorar el desempeño de los estudiantes en educación superior.

- Implementar el ambiente tecnológico basado en aplicación de estrategias como medio para lograr un mejor desempeño académico de los estudiantes.

- Validar el modelo a partir de los efectos de la aplicación de estrategias mediadas con TIC, en relación con los niveles de comprensión y el desempeño de los grupos sujeto de estudio.

Es esta la razón que lleva a formular la hipótesis: la aplicación de estrategias mediadas con TIC produce diferencias significativas en el desempeño de los estudiantes del grupo experimental respecto al desempeño del grupo control.

\section{Metodología}

Diseño del curso

El diseño se hizo con base en los postulados del alineamiento constructivo. El enfoque constructivista y el aprendizaje significativo dan soporte teórico y metodológico a las características y estrategias que facilitan el desarrollo de competencias, para lograr un aprendizaje autónomo y un trabajo independiente; fundamentos vitales en los procesos de aprendizaje. La teoría del alineamiento constructivo, propuesta por Biggs (2005, p. 324) para el diseño de la enseñanza se basa en el principio del constructivismo: aprendizaje y alineamiento en la enseñanza. El alineamiento constructivo consiste en alinear el método y la evaluación de la enseñanza con las actividades de aprendizaje establecidas en los objetivos, de manera que todos los aspectos de este sistema estén de acuerdo en apoyar el aprendizaje del estudiante. El curso se operacionaliza mediante el Modelo 3P, empleando la Taxonomía Solo y desarrollando los enfoques de aprendizaje.

\section{Enfoques de aprendizaje}

A partir de la investigación de Marton y Saljö (1976a y1976b, citados en Hernández Pina et al. 2005, p. 124), orientada a conocer el grado de comprensión de los alumnos al leer-estudiar un texto y comprender las diferencias individuales evidenciadas por los alumnos en su proceso de aprendizaje al abordar los contenidos, los cuales determinan la orientación de la tarea, los autores acuñan los términos Enfoque Profundo y Enfoque Superficial del Aprendizaje para referirse a las dos formas que adoptan los estudiantes al procesar información. El término superficial se refiere al procesamiento dirigido al texto en sí mismo, indicando una concepción reproductiva del aprendizaje. El término profundo se refiere a alumnos centrados en la comprensión del texto, mostrando mayor interés por el significado. Los investigadores apostaron por la hipótesis que planteaba que estas dos formas cualitativas de abordar el material reflejan diferencias en los niveles de comprensión alcanzados por el alumno.

Los enfoques superficial y profundo son dos formas de relacionarse, los estudiantes con un ambiente de enseñanza y aprendizaje, pero no son características fijas de éstos, son respuestas de naturaleza racional a sus ambientes educativos: Por tanto, se evalúan los niveles de complejidad en los resultados del 
aprendizaje, mediante la aplicación de la propuesta de Biggs y Collins (1982, p. 231). Se concreta en la Taxonomía Solo, una manera sistemática de describir el aumento de la complejidad de la actuación de un aprendiz cuando domina tareas académicas. Ésta se utiliza para definir objetivos curriculares, que describan dónde deben estar operando los estudiantes y para evaluar los resultados, de manera que sepamos en qué nivel se están desenvolviendo. Las propiedades de Solo evalúan en forma objetiva la calidad del resultado, determinan el uso de componentes de manera integrada. Las características se basan en el progreso en el aprendizaje; en el hecho de que los estudiantes avanzan en niveles de complejidad crecientes, y cómo van relacionando sus respuestas en aspectos abstractos de las tareas.

Tabla 1: Niveles integrados de Solo.

\section{Nivel preestructural: respuestas centradas en aspectos irrelevantes de la propuesta de trabajo, con respuestas evasivas o tautologías del enunciado \\ II. Nivel uniestructural; respuestas que contienen datos informativos y obvios, los cuales han sido extraídos directamente del enunciado. \\ III. Nivel multiestructural: respuestas que requieren la utilización de dos o más informaciones del enunciado, las cuales, siendo obtenidas directamente de éste, son analizadas separadamente, no de forma interrelacionada. \\ IV. Nivel relacional: respuestas extraídas tras el análisis de los datos del problema, integrando la información en un todo comprensivo. Los resultados se organizan formando una estructura.}

V. Nivel abstracción expandida: respuestas que manifiestan la utilización de un principio general y abstracto que puede ser inferido a partir de los datos del problema y es generalizable a otros contextos.

La principal línea divisoria está entre los niveles III y IV. En los niveles IV y V, las respuestas envuelven evidencias de comprensión, en el sentido de integrar y estructurar las partes del material. Las categorías oscilan entre respuestas no estructuradas, que usan información irrelevante, y abstracciones de alto nivel, que usan la información para formular hipótesis. Solo sitúa las respuestas en categorías predeterminadas y jerarquizadas, de acuerdo con su calidad.
Biggs y Collins (1982) aportan ejemplos aplicables a diversas disciplinas, mostrando la manera en que los cinco niveles de la taxonomía se localizan en los productos alcanzados por los estudiantes, tras la realización de tareas. El nivel preestructural es simple, indica que no ha habido comprensión. En los niveles uniestructural y multiestructural, se concibe comprender como un incremento cuantitativo de los resultados alcanzados. Las respuestas construidas en torno al más alto nivel incluyen los más bajos y un poco más. El poco más, en el caso del nivel relacional sobre el multiestructural, implica una reestructuración conceptual de los componentes y el reconocimiento de un sistema que los integra. El último nivel supone que el alumno se implica profundamente con el material, relaciona los contenidos con los conocimientos previos y generaliza sobre aspectos no presentados en el material original.

\section{El modelo teórico 3P}

La necesidad de establecer un modelo teórico incluyendo las variables mediadoras del proceso de estudio llevó a Biggs (1991, 1993 y 1996b, citado por Hernández Pina et al., 2005, p. 128) a adoptar el Modelo 3P, el cual señala tres puntos en los que sitúa los factores relacionados con el aprendizaje: Pronóstico o presagio, antes de que se produzca el aprendizaje; Proceso, durante el aprendizaje, y Producto o resultado del aprendizaje. Las variables del ámbito personal e institucional, (factores de presagio) relacionadas con el alumno: concepciones de aprendizaje, conocimientos previos, motivación, habilidades, modos preferentes de aprender, hábitos de trabajo, valores, expectativas, se engloban con las variables del contexto de enseñanza: estructura del curso, contenidos curriculares, métodos de enseñanza, clima de la clase y evaluación. Las variables mediadoras, los efectos de los procesos metacognitivos y los enfoques de aprendizaje (factores de proceso) son consecuencia de la interacción entre los factores personales y los contextuales, y se refieren al modo en que los estudiantes abordan las tareas de aprendizaje, adoptando un enfoque de aprendizaje con motivo y estrategia. El resultado del aprendizaje (factores de producto) -cuantitativos, cualitativos, 
afectivos- son determinados principalmente por la combinación de los dos anteriores.

Gráfica 1: Modelo 3P de enseñanza y aprendizaje.

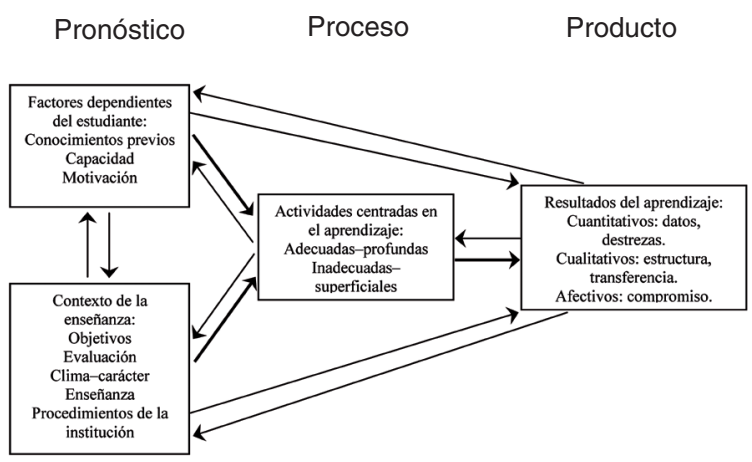

Fuente: Biggs, 2005.

El modelo es un sistema integrado e interactivo, configurado en torno a tres componentes del aprendizaje:

Los factores de presagio apoyan las variables de proceso o mediadoras, los enfoques de aprendizaje describen la dinámica de enseñanza-aprendizaje, que ocurre durante la interacción en clase y de la cual resulta la fase producto, muchas veces sinónimo de resultados Biggs (1993 y 1996, citado por Hernández Pina et al., 2005, p. 148).

Los estudiantes interpretan su contexto a la luz de sus propias preconcepciones y motivaciones, desarrollando una actividad metacognitiva centrada en el proceso de aprendizaje. De esta actividad de meta-aprendizaje resulta la preferencia por un determinado enfoque, que influirá en el resultado final (p. 156).

Los estudiantes no son profundos o superficiales, más bien adoptan el enfoque de aprendizaje que consideran apropiado a las circunstancias y les permite llevar a cabo las tareas académicas lo más cómodamente posible. Las variables de producto corresponde $\mathrm{n}$ a los resultados que pueden ser descritos cuantitativamente (cuánto he aprendido), cualitativamente (qué tan bien he aprendido). En el modelo, el resultado del aprendizaje está determinado por factores que interactúan entre sí. La dirección general de los efectos está marcada por las flechas gruesas: los factores de pronóstico del estudiante y de la enseñanza determinan conjuntamente el enfoque que emplee el estudiante en una tarea dada, lo que, a su vez, determina el resultado. La flechas finas conectan todo con todo, porque los componentes constituyen un sistema (Biggs, 2005).

Los componentes de este sistema derivan de los estudiantes y del contexto de la enseñanza, determinando los procesos cognitivos que con mayor probabilidad utilizarán los estudiantes, quienes, a su vez, determinan los detalles y la estructura inherentes a los resultados del aprendizaje.

Del modelo se infiere que los estudiantes interpretan su contexto de aprendizaje a la luz de sus preconcepciones y motivaciones, desarrollando una actividad metacognitiva centrada en el proceso de aprendizaje. Este modelo describe cómo los individuos difieren en su contexto de enseñanza (enfoque preferencial), cómo manejan tareas específicas (enfoque adoptado), o cómo los contextos de enseñanza difieren unos de otros (enfoque contextual) (Hernández Pina et al., 2005, citando a Biggs et al., 2001).

El Modelo 3P encuadra los objetivos perseguidos en la investigación; como marco teórico de referencia para promover el aprendizaje, mediante las relaciones entre lo que piensan y hacen y la naturaleza de sus resultados de aprendizaje. Permite comprobar la posible relación lineal entre los factores de presagio (concepciones de aprendizaje), factores de proceso (enfoques de aprendizaje) y los factores de producto (desempeño académico) y la interacción entre los componentes que forman este sistema. De esta forma, se comprueba la posible relación entre las concepciones de aprendizaje (presagio) y el desempeño académico (producto), mediatizados por los enfoques de aprendizaje (proceso).

\section{Contenidos}

El contenido está vinculado con los métodos y técnicas de trabajo de la Física, mediante conceptos, leyes, principios, teorías, así como con las actividades necesarias en las relaciones con el objeto de estudio. Está sugerido desde el Departamento de Física y Electrónica de la Universidad de Córdo- 
ba, se organizó a partir de un modelo de enfoque sistémico estructural-funcional. Es presentado de manera secuencial y enmarcado en un contexto que permite su comprensión. Se organizó considerando el plan general: identificación, justificación, objetivos del curso, sistema de competencias, unidades de aprendizaje con sus respectivos objetivos, contenidos y actividades, metodología y evaluación; se complementó con los planes por competencias de cada unidad, permitiendo conocer las condiciones, a partir de las cuales se debe llevar a cabo el proceso de aprendizaje: competencias y niveles de comprensión por alcanzar; objetivos que se deben desarrollar; contenidos declarativos (teóricos), procedimentales (aplicación) y actitudinales (valoración, motivación) que se deben gestionar; actividades a realizar en los espacios de docencia directa y trabajo independiente; metodología a seguir; indicadores evaluadote evaluación y evidencias a presentar al finalizar su proceso de aprendizaje. Además de talleres y guías que contienen lo relacionado con la parte procedimental del curso.

\section{Estructura}

En un proceso educativo, el trabajo depende de la estructura del texto, del discurso. Si éste no existe o lleva al estudiante a cualquier dirección, no puede aspirarse a que aprenda algo; por tanto, se presenta el sitio web propuesto:

Tabla 2: Propuesta para la estructuración del curso mediante el sitio web.

\begin{tabular}{|l|l|}
\hline Título & $\begin{array}{l}\text { Describe el tema objeto de aprendizaje en } \\
\text { forma sintética. }\end{array}$ \\
\hline Presentación & $\begin{array}{l}\text { Se hace una descripción del curso y de } \\
\text { los recursos a utilizar para la revisión } \\
\text { de contenidos, desarrollo de actividades } \\
\text { y comunicación con los demás } \\
\text { participantes. Se invita a participar de } \\
\text { la construcción de aprendizajes desde } \\
\text { cualquier lugar, de acuerdo con sus ritmos } \\
\text { de aprendizaje y tiempo disponible. }\end{array}$ \\
\hline
\end{tabular}

\begin{tabular}{|c|c|}
\hline $\begin{array}{l}\text { Planeación y } \\
\text { organización } \\
\text { del curso }\end{array}$ & $\begin{array}{l}\text { Plan del curso: contiene y describe los } \\
\text { aspectos generales y la secuencia de } \\
\text { los contenidos a observar durante el } \\
\text { desarrollo del curso. } \\
\text { Planes por competencias de cada unidad } \\
\text { de aprendizaje: diseñados teniendo en } \\
\text { cuenta el alineamiento constructivo, se } \\
\text { describe la actuación del estudiante en } \\
\text { cada unidad, al reconocer en ellos las } \\
\text { competencias y niveles de comprensión } \\
\text { que debe alcanzar, los objetivos de } \\
\text { aprendizaje que debe desarrollar, los } \\
\text { contenidos declarativos, procedimentales } \\
\text { y actitudinales que debe gestionar, las } \\
\text { actividades a realizar en los espacios de } \\
\text { docencia directa y trabajo independiente; } \\
\text { la metodología a seguir, los indicadores } \\
\text { con que será evaluado y las evidencias } \\
\text { que debe presentar luego de finalizado su } \\
\text { proceso de aprendizaje. }\end{array}$ \\
\hline Contenidos & $\begin{array}{l}\text { Los contenidos revisados desde este } \\
\text { espacio son sugeridos por docentes y } \\
\text { estudiantes y su criterio de selección } \\
\text { tiene que ver con la calidad, relevancia, } \\
\text { pertinencia y actualidad; características } \\
\text { que permiten al estudiante ampliar su } \\
\text { conocimiento y mejorar su comprensión. } \\
\text { Para el desarrollo del curso, los docentes y } \\
\text { los estudiantes, además de la bibliografía } \\
\text { sugerida, utilizan el curso Física con } \\
\text { ordenador, el cual es interactivo, del autor } \\
\text { Ángel Franco García: http://www.sc.ehu. } \\
\text { es/sbweb/fisica/default.htm } \\
\text { Este curso de física general trata desde } \\
\text { conceptos simples como el movimiento } \\
\text { rectilíneo hasta otros más complejos como } \\
\text { las bandas de energía de los sólidos, } \\
\text { cuya interactividad se logra mediante los } \\
586 \text { applets insertados en sus páginas, } \\
\text { son simulaciones de sistemas físicos, } \\
\text { prácticas de laboratorio, experiencias } \\
\text { de gran relevancia histórica, problemas } \\
\text { interactivos, problemas-juego, etc. }\end{array}$ \\
\hline $\begin{array}{l}\text { Actividades y } \\
\text { estrategias }\end{array}$ & $\begin{array}{l}\text { Las actividades y estrategias para que el } \\
\text { alumnos obtenga un mejor aprendizaje de } \\
\text { los fenómenos físicos está dada por: } \\
\text { Talleres: contiene ejercicios para el } \\
\text { afianzamiento de los temas vistos. } \\
\text { Guías: son una serie de acciones } \\
\text { propuestas por el docente para que } \\
\text { el estudiante pueda llevar a cabo un } \\
\text { procedimiento observable y medible para } \\
\text { el resultado. } \\
\text { Simulaciones: alternativa metodológica } \\
\text { de solución a la descontextualización del } \\
\text { aprendizaje, descrita en el planteamiento } \\
\text { del problema. Permite que puedan } \\
\text { resolver sus inquietudes, tener una mayor } \\
\text { comprensión de los fenómenos al ser } \\
\text { recreados de manera artificial y manipular } \\
\text { las variables implicadas. }\end{array}$ \\
\hline
\end{tabular}




\begin{tabular}{|c|c|}
\hline Foros & $\begin{array}{l}\text { Comunicación asíncrona en la que se } \\
\text { propicia el debate, la concertación } \\
\text { y el consenso de ideas. Permite } \\
\text { publicar su mensaje en cualquier } \\
\text { momento, quedando visible para que } \\
\text { otros compañeros y docentes, puedan } \\
\text { leerlo y contestar. Asimismo, permite } \\
\text { mantener la comunicación entre } \\
\text { estudiantes y docentes, sin necesidad } \\
\text { de coincidir en horarios de encuentro en } \\
\text { la red, superando las limitaciones de la } \\
\text { comunicación sincrónica y dilatando en } \\
\text { el tiempo los ciclos de interacción, lo cual } \\
\text { favorece la reflexión y la madurez de los } \\
\text { mensajes. } \\
\text { En el curso contaremos con dos } \\
\text { categorías: } \\
\text { 1. Foro general: se encuentra en la } \\
\text { sección inicial del curso, que es aquella } \\
\text { que, por defecto, siempre aparece al } \\
\text { principio. } \\
2 \text {. Foros de aprendizaje: aparecen al final } \\
\text { de cada unidad de aprendizaje, diseñados } \\
\text { para tratar los temas e inquietudes de } \\
\text { dicha unidad. }\end{array}$ \\
\hline $\begin{array}{l}\text { Glosario de } \\
\text { términos }\end{array}$ & $\begin{array}{l}\text { Permite a docentes y estudiantes } \\
\text { mantener una lista de definiciones } \\
\text { relacionadas con el tema de la Física. } \\
\text { El docente presenta los conceptos clave } \\
\text { del curso, para ser comentados por } \\
\text { los alumnos. A los estudiantes se les } \\
\text { permite experimentar una herramienta } \\
\text { colaborativa que ayuda en su desarrollo } \\
\text { (proceso) y como referencia de consulta } \\
\text { (producto final). }\end{array}$ \\
\hline Evaluación & $\begin{array}{l}\text { Instrumentos que facilitan la evaluación } \\
\text { por competencias del desempeño, } \\
\text { conocimientos y productos. }\end{array}$ \\
\hline
\end{tabular}

Así se planteó la estructura del curso, al cual se puede acceder en la dirección http://www.edunexos.edu. co/genius/. La temática organizada de este modo ofrece caminos para el desarrollo competente del aprendizaje; permite secuencias, encontrar correspondencia entre lo anterior y lo posterior, acercarse a un tema para verlo desde distintos ángulos.

\section{Diseño metodológico}

Para alcanzar los objetivos, responder a la pregunta de investigación y someter a prueba la hipótesis formulada, se seleccionó un diseño cuasiexperimental, que permitió la intervención sobre grupos intactos. Se eligió el modelo preprueba- posprueba con grupo control, cuyo esquema es:
Tabla 3: Esquema del modelo preprueba-posprueba.

\begin{tabular}{|c|c|c|c|}
\hline Grupos & $\begin{array}{c}\text { Medida de la } \\
\text { preprueba }\end{array}$ & Tratamiento & $\begin{array}{c}\text { Medida de } \\
\text { la posprueba }\end{array}$ \\
\hline Experimental & $0_{1}$ & $X$ & $0_{2}$ \\
\hline Control & $0_{1}$ & -- & $0_{2}$ \\
\hline
\end{tabular}

Fuente: (Hernández et al., 1999, p. 29).

\section{Variables}

Se tomó como variable dependiente el desempeño académico y como independiente el ambiente de aprendizaje. Se definieron dimensiones e indicadores, que resaltan lo fundamental de cada variable, como se observa: En la Tabla 4.

\section{Población sujeto de estudio}

La población está conformada por ciento diez estudiantes matriculados en el segundo periodo académico de 2007 en el curso Física I (Fundamentos de mecánica), correspondiente al segundo semestre del Programa de Ingeniería Industrial de la Universidad de Córdoba. Se conformaron dos grupos intactos de cincuenta y cuatro y cincuenta y seis estudiantes, respectivamente, cada uno eligió de manera voluntaria uno de los dos horarios, propuestos para ese semestre académico. Para la validez de la presente investigación, se estableció la equivalencia de los grupos, a partir de los criterios que se describen a continuación:En la Tabla 5.

En el grupo experimental, se aplicaron las estrategias basadas en TIC para el desarrollo del desempeño académico, y en el grupo control, se usó el programa construido por el Departamento de Física para Ingenierías, el cual se viene aplicando con la metodología tradicional: clases magistrales, realización de prácticas, sesiones de solución de problemas y evaluaciones escritas. 
Tabla 4: Variables.

\begin{tabular}{|c|c|c|c|}
\hline Variable dependiente & Definición conceptual & Dimensión & Indicadores \\
\hline \multirow{3}{*}{ Desempeño académico } & \multirow{3}{*}{$\begin{array}{l}\text { Se refiere a la calidad del aprendizaje } \\
\text { evidenciada en motivación, valor de } \\
\text { la tarea, autoconcepto cognitivo y, en } \\
\text { general, comprende la gestión de sus } \\
\text { aprendizajes y la valoración final. }\end{array}$} & $\begin{array}{l}\text { Enfoque de } \\
\text { aprendizaje }\end{array}$ & $\begin{array}{l}\text { Enfoque profundo } \\
\text { - Motivación profunda. } \\
\text { - Estrategia profunda. } \\
\text { Enfoque superficial } \\
\text { - Motivación superficial. } \\
\text { - Estrategia superficial. }\end{array}$ \\
\hline & & $\begin{array}{l}\text { Niveles de } \\
\text { comprensión }\end{array}$ & $\begin{array}{l}\text {-Preestructural. } \\
\text { - Uniestructural. } \\
\text { - Multiestructural. } \\
\text { - Relacional. } \\
\text { - Abstracción expandida. }\end{array}$ \\
\hline & & $\begin{array}{l}\text { Resultados del } \\
\text { aprendizaje }\end{array}$ & $\begin{array}{l}\text { - Desempeño. } \\
\text { - Conocimiento. } \\
\text { - Productos. }\end{array}$ \\
\hline Variable independiente & Definición conceptual & Dimensión & Indicadores \\
\hline \multirow[b]{2}{*}{ Ambiente de aprendizaje } & \multirow[b]{2}{*}{$\begin{array}{l}\text { Circunstancias que se disponen y las } \\
\text { estrategias que se usan para promover } \\
\text { el desarrollo del aprendizaje. }\end{array}$} & $\begin{array}{l}\text { Percepción de } \\
\text { contextos de } \\
\text { enseñanza. }\end{array}$ & $\begin{array}{l}\text { Centrado en: } \\
\text { El estudiante. } \\
\text { El docente. } \\
\text { Los métodos } \\
\text { La integración de todos los elementos anteriores. }\end{array}$ \\
\hline & & $\begin{array}{l}\text { Estrategias mediadas } \\
\text { con TIC.. }\end{array}$ & $\begin{array}{l}\text { Sitio web: } \\
\text { - Funcionalidad (eficacia, facilidad y versatilidad). } \\
\text { - Técnica y estética (calidad del entorno } \\
\text { audiovisual, de los contenidos, navegación e } \\
\text { interacción y originalidad). } \\
\text { - Pedagogía (capacidad de motivación, adecuación } \\
\text { a los usuarios y a su ritmo de trabajo). } \\
\text { El docente: } \\
\text { - Organización y acompañamiento del trabajo } \\
\text { independiente. } \\
\text { - Uso de estrategias. } \\
\text { Actitud del estudiante: } \\
\text { - Efectos cognitivos (habilidades, nivel de } \\
\text { desempeño, estrategias de aprendizaje que utiliza). } \\
\text { - Efectos psicológicos (capacidad de motivación y } \\
\text { nivel de autonomía). } \\
\text { - Efecto comunicativo (interacción con el profesor). }\end{array}$ \\
\hline
\end{tabular}

Tabla 5: Criterios para la conformación de grupos.

\begin{tabular}{|l|l|l|}
\hline Criterios & Grupo experimental & Grupo control \\
\hline Número de estudiantes & $54(49 \%)$ & $56(51 \%)$ \\
\hline Género & $30 \mathrm{~h}, 24 \mathrm{~m}(55,6 \%, 44,4 \%)$ & $35 \mathrm{~h}, 21 \mathrm{~m}(62,5 \%, 37,5 \%)$ \\
\hline Promedio puntajes Icfes & 55,25 & 56,14 \\
\hline Nivel socio-económico & Estratos 2,3 y 4 & Estratos 2,3 y 4 \\
\hline Promedio de edad & 17,8 años & 17,5 años \\
\hline Número de repitentes & 4 & 5 \\
\hline Horario & Diurno & Diurno \\
\hline
\end{tabular}




\section{Instrumentos para la recolección de la información}

Para determinar enfoques y evaluar estrategias de aprendizaje, se utilizó el Revised Two Factor Study Process Questionnaire R-SPQ-2F4, de Biggs et al. (2001), validado en investigaciones realizadas en universidades españolas, norteamericanas y latinoamericanas. Consta de veinte proposiciones que describen la actitud hacia el estudio; la medición se hace por medio de escala tipo Likert de cinco puntos, en la cual el estudiante marca acuerdo o desacuerdo, según la proposición se acerque o aleje de la representación de sus aptitudes hacia el estudio. De las veinte proposiciones, diez contribuyen al factor profundo y diez al superficial. En cada factor se distinguen dos subescalas: motivo y estrategia, conformadas por cinco ítems. Tiene dos escalas principales: Enfoque Profundo, ep, y Enfoque Superficial, es, con cuatro subescalas: Estrategia Superficial, es; Motivación Superficial, ms; Estrategia Profunda, ep, y Motivación Profunda, mp.

El Factor Superficial se conforma con la suma de Motivación Superficial y Estrategia Superficial (ms+es=ts). El Factor Profundo por la suma de Motivación Profunda y Estrategia Profunda $(\mathrm{mp}+\mathrm{ep}=\mathrm{tp})$. Para la confiabilidad de los ítems, se utilizó Alpha de Cronbach, con el programa SPSS 10.0 y se aplicó a dieciocho estudiantes:

\begin{tabular}{|c|}
\hline Reliability analysis - scale ( alpha) \\
\hline Reliability Coefficients \\
N of Cases $=18,0 \mathrm{~N}$ of Items $=20$ \\
Alpha $=, 6190$
\end{tabular}

El coeficiente Alpha de Cronbach toma valores entre cero y uno; cero significa confiabilidad nula y uno confiabilidad total. El anterior resultado es confiable, se acerca a uno y se aleja de cero, indicando correlación lineal entre los ítems.

Para evaluar los niveles de comprensión en los grupos, se diseñó una prueba a partir de una lectura de temas relacionados con el curso, en la

4 Cuestionario de procesos de estudio-dos factores. cual se solicita describir lo positivo, lo negativo, los interrogantes y comentarios.

La confiabilidad del instrumento se realizó con el modelo Alpha de Cronbach, mediante el programa SPSS 10.0, aplicado a veinte estudiantes del curso:

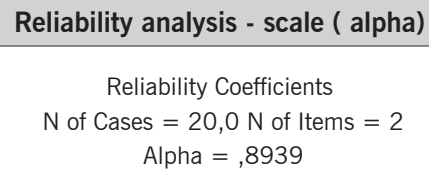

El resultado es confiable, indicando, alta correlación lineal entre los ítems.

Para evaluar los niveles de comprensión en los dos grupos, se diseñó una prueba a partir de una lectura de temas relacionados con el curso, se solicita describir lo positivo, lo negativo, los interrogantes y comentarios. El instrumento fue evaluado teniendo en cuenta los indicadores de cada nivel de comprensión, integrado en Solo, ajustado desde la teoría y el juicio de expertos. Su confiabilidad se determinó con el Alpha de Cronbach, mediante el SPSS 10.0 y se aplicó a nueve docentes del Programa, se midió sobre los veintidós ítems de la prueba:

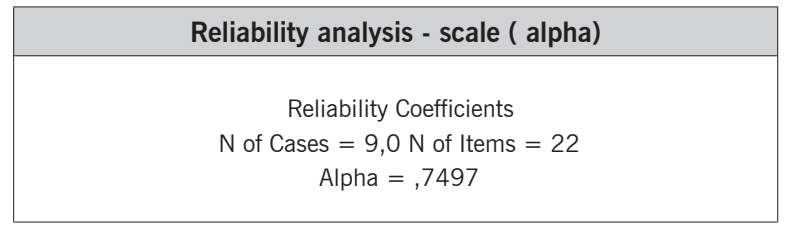

El resultado es confiable, indicando correlación lineal entre ítems.

Para evaluar los efectos de la aplicación de las estrategias mediadas con TIC, se diseñó una prueba de receptividad con escalonamiento tipo Likert, consistente en un conjunto de veintitrés afirmaciones, frente a las cuales se midió la reacción de los estudiantes del grupo experimental. La prueba explora la percepción respecto a la estrategia pedagógica: en general, frente al docente y frente los recursos.

El instrumento fue revisado por expertos y su confiabilidad se determinó con el Alpha de Cronbach, mediante el SPSS 10.0 y se aplicó a diez estudiantes del curso: 


\begin{tabular}{|c|}
\hline Reliability analysis - scale ( alpha) \\
\hline Reliability Coefficients \\
$\mathrm{N}$ of Cases $=10,0 \mathrm{~N}$ of Items $=23$ \\
Alpha $=, 7465$ \\
\hline
\end{tabular}

El resultado es confiable e indica alta correlación lineal entre los ítems.

\section{Resultados}

\section{Resultados de la preprueba}

\section{Distribución de enfoques de aprendizaje}

La preprueba para identificar enfoques y estrategias de aprendizaje, contextos de enseñanza o condiciones internas y externas al iniciar el proceso arrojó los resultados siguientes:

Tabla 6: resultados grupo experimental.

Enfoque de aprendizaje

\begin{tabular}{|ll|r|r|r|r|}
\hline & Frecuencia & Porcentaje & $\begin{array}{c}\text { Porcentaje } \\
\text { Válido }\end{array}$ & $\begin{array}{c}\text { Porcentaje } \\
\text { Acumulado }\end{array}$ \\
\hline Válidos & Profundo & 50 & 89,3 & 89,3 & 89,3 \\
& Superficial & 6 & 10,7 & 10,7 & 100,0 \\
& Total & 56 & 100,0 & 100,0 & \\
\hline
\end{tabular}

Tabla 7: resultados grupo control.

\begin{tabular}{|c|c|c|c|c|c|}
\hline \multicolumn{6}{|c|}{ Enfoque de aprendizaje } \\
\hline & & Frecuencia & Porcentaje & $\begin{array}{l}\text { Porcentaje } \\
\text { Válido }\end{array}$ & $\begin{array}{l}\text { Porcentaje } \\
\text { Acumulado }\end{array}$ \\
\hline \multirow[t]{3}{*}{ Válidos } & Profundo & 47 & 87,0 & 87,0 & 87,0 \\
\hline & Superficial & 7 & 13,0 & 13,0 & 100,0 \\
\hline & Total & 54 & 100,0 & 100,0 & \\
\hline
\end{tabular}

En ambos grupos, la mayoría presenta enfoque profundo: $87,0 \%$ experimental y $89,3 \%$ control y $13,0 \%$ y $10,7 \%$ enfoque superficial, siendo similares los grupos en esta dimensión. La prueba de hipótesis para la diferencia de dos proporciones, con $\mathrm{p}_{1}$ y $\mathrm{p}_{2}$ las proporciones de estudiantes en los grupos experimental y control, respectivamente, con $\mathrm{H}_{0}$ : $\mathrm{p}_{1}=\mathrm{p}_{2} \mathrm{Vs} \mathrm{H}_{1}: \mathrm{p}_{1} \neq \mathrm{p}_{2}$, estableciendo que no existe diferencia significativa si $\mathrm{p}<0,05$ o altamente significativa si $\mathrm{p}<0,01$. La comparación de proporciones para la distribución de enfoques de aprendizaje de los grupos arroja lo siguiente:

Tabla 8: Comparación de proporciones para la distribución de enfoques de aprendizaje.

\begin{tabular}{|c|c|}
\hline Enfoque & P-Valor \\
\hline Profundo & $0,71 \mathrm{~ns}^{*}$ \\
Superficial & $0,71 \mathrm{~ns}$ \\
\hline
\end{tabular}

*ns: no hay significancia estadística.

Los grupos no presentan diferencias significativas en la preprueba, en lo relacionado con enfoques de aprendizaje, indicando que son equivalentes.

\section{Distribución de niveles de comprensión}

Tabla 9: Distribución de niveles de comprensión grupo experimental.

\begin{tabular}{|l|r|r|r|r|}
\hline & Niveles de Comprensión \\
\hline & Frecuencia & Porcentaje & $\begin{array}{c}\text { Porcentaje } \\
\text { Válido }\end{array}$ & $\begin{array}{c}\text { Porcentaje } \\
\text { Acumulado }\end{array}$ \\
\hline Válidos Uniestructural & 27 & 50,0 & 50,0 & 50,0 \\
Multiestructural & 18 & 33,0 & 33,0 & 83,3 \\
Relacional & 7 & 13,0 & 13,0 & 96,3 \\
Abstracto Ampliado & 2 & 3,7 & 3,7 & 100,0 \\
Total & 54 & 100,0 & 100,0 & \\
\hline
\end{tabular}

Tabla 10: Distribución de niveles de comprensión grupo control.

\begin{tabular}{|l|r|r|r|r|}
\hline & Niveles de Comprensión \\
\hline & Frecuencia & Porcentaje & $\begin{array}{c}\text { Porcentaje } \\
\text { Válido }\end{array}$ & $\begin{array}{c}\text { Porcentaje } \\
\text { Acumulado }\end{array}$ \\
\hline Válidos Preestructural & 1 & 1,8 & 1,8 & 1,8 \\
Uniestructural & 29 & 51,8 & 51,8 & 53,6 \\
Multiestructural & 18 & 32,1 & 32,1 & 85,7 \\
Relacional & 7 & 12,5 & 12,5 & 98,2 \\
Abstracto Amplio & 1 & 1,8 & 1,8 & 100,0 \\
Total & 56 & 100,0 & 100,0 & \\
\hline
\end{tabular}

En el nivel preestructural se ubica un estudiante del grupo control (1,8\%). En el uniestructural se ubica la mayoría de ambos grupos, experimental y control, el 50,0\% y 51,8\% respectivamente; en el 
multiestructural se ubica el $33,3 \%$ y $32,1 \%$, en el relacional el $13,0 \%$ y $12,5 \%$; y en el abstracto ampliado el $3,7 \%$ y $1,8 \%$, respectivamente. La prueba de hipótesis para la diferencia de proporciones, con $\mathrm{p}_{1}$ y $\mathrm{p}_{2}$ los promedios en los grupos experimental y control respectivamente, considerando $\mathrm{H}_{0}: \mathrm{p}_{1}=\mathrm{p}_{2}$ Vs $\mathrm{H}_{1}: \mathrm{p}_{1} \neq \mathrm{p}_{2}$, estableciendo que no existe diferencia significativa si $\mathrm{p}<0,05$ o altamente significativa si $\mathrm{p}<0,01$, arroja los resultados:

Tabla 11: Resultados de la prueba de hipótesis para la diferencia de proporciones.

\begin{tabular}{|c|c|}
\hline Nivel de comprensión & P-Valor \\
\hline Preestructural & $0,32 \mathrm{~ns}^{*}$ \\
\hline Uniestructural & $0,86 \mathrm{~ns}$ \\
\hline Multiestructural & $0,90 \mathrm{~ns}$ \\
\hline Relacional & $0,94 \mathrm{~ns}$ \\
\hline Abstracto ampliado & $0,56 \mathrm{~ns}$ \\
\hline
\end{tabular}

*ns: no significancia estadística.

Los grupos no presentan diferencias significativas en niveles de comprensión, ya que todos poseen un p-valor mayor a 0,05. Es decir, son equivalentes.

\section{Resultados de las posprueba Análisis descriptivo}

Para evaluar el modelo, se tuvieron en cuenta dos aspectos: desempeño académico de los estudiantes (notas parciales 1, 2 y 3 y definitiva) y la prueba de receptividad, relacionada con el desarrollo de la estrategia. La valoración de los productos se realizó de acuerdo con el Reglamento Académico Estudiantil, RAE. Se asignó un valor de 30\% a informes de laboratorio, $30 \%$ a talleres y $40 \%$ a exámenes escritos. Se organizaron las notas parciales y definitivas en cinco rangos, comparables con los niveles de comprensión de la Taxonomía Solo, como se muestra a continuación:

Tabla 12: Comparación del rango de notas con los niveles de comprensión de la Taxonomía Solo.

\begin{tabular}{|c|c|}
\hline Nivel de comprensión & Rango de notas \\
\hline Preestructural & $0,0-1,5$ \\
\hline Uniestructural & $1,6-2,9$ \\
\hline
\end{tabular}

\begin{tabular}{|c|c|}
\hline Multiestructural & $3,0-3,4$ \\
\hline Relacional & $3,5-3,9$ \\
\hline Abstracto ampliado & 4,0 ó más \\
\hline
\end{tabular}

\section{Resultados del primer parcial}

Tabla 13: Resultados del primer parcial grupo experimental.

\begin{tabular}{|} 
Rangos de desempeño primer parcial \\
\begin{tabular}{|r|r|r|r|r|}
\hline & Frecuencia & Porcentaje & $\begin{array}{c}\text { Porcentaje } \\
\text { Válido }\end{array}$ & $\begin{array}{r}\text { Porcentaje } \\
\text { Acumulado }\end{array}$ \\
\hline Válidos $1,6-2,9$ & 22 & 44,0 & 44,0 & 44,0 \\
$3,0-3,4$ & 22 & 44,0 & 44,0 & 88,0 \\
$3,5-3,9$ & 3 & 6,0 & 6,0 & 94,0 \\
$4,0-5,0$ & 3 & 6,0 & 6,0 & 100,0 \\
Total & 50 & 100,0 & 100,0 & \\
\hline
\end{tabular}
\end{tabular}

Tabla 14: Resultados del primer parcial grupo control.

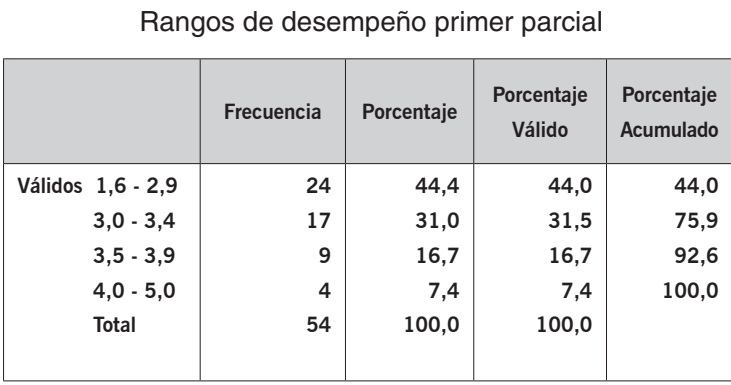

En el primer parcial, hubo bajos niveles de desempeño por parte de los dos grupos: el 44,0\% y $44,4 \%$ en el experimental y control, respectivamente, obtienen notas inferiores a 3,0, ubicándose en el nivel uniestructural. En el rango entre 3,0 y 3,4, nivel multiestructural, el experimental obtiene el $44 \%$, mientras que el control $31,5 \%$. Para el rango entre 3,5 y 3,9, nivel relacional, el 6\% del grupo experimental y $16,7 \%$ del grupo control y para el rango superior a 4,0 , abstracto ampliado, el grupo experimental tuvo un $6 \%$, mientras que en el grupo control $7,4 \%$. Es necesario resaltar que hay un mayor porcentaje de estudiantes del grupo control ubicados en los niveles relacional y abstracto ampliado. Niveles que en la Taxonomía Solo se consideran superiores. 


\section{Resultados del segundo parcial}

Tabla 15: Resultados del segundo parcial grupo experimental.

\begin{tabular}{|c|r|r|r|r|}
\hline & Frecuencia & Porcentaje & $\begin{array}{c}\text { Porcentaje } \\
\text { Válido }\end{array}$ & $\begin{array}{c}\text { Porcentaje } \\
\text { Acumulado }\end{array}$ \\
\hline & 20 & 40,0 & 40,0 & 40,0 \\
\hline Válidos de desempeño segundo parcial \\
\hline $1,6-2,9$ & 15 & 30,0 & 30,0 & 70,0 \\
$3,0-3,4$ & 12 & 24,0 & 24,0 & 94,0 \\
$3,5-3,9$ & 3 & 6,0 & 6,0 & 100,0 \\
$4,0-5,0$ & 50 & 100,0 & 100,0 & \\
Total & & & & \\
\hline
\end{tabular}

Tabla 16: Resultados del primer parcial grupo control.

Rangos de desempeño segundo parcial

\begin{tabular}{|c|r|r|r|r|}
\hline & Frecuencia & Porcentaje & $\begin{array}{c}\text { Porcentaje } \\
\text { Válido }\end{array}$ & $\begin{array}{c}\text { Porcentaje } \\
\text { Acumulado }\end{array}$ \\
\hline Válidos $0,0-1,5$ & 1 & 1,9 & 1,9 & 1,9 \\
$1,6-2,9$ & 29 & 53,7 & 53,7 & 55,6 \\
$3,0-3,4$ & 12 & 22,2 & 22,2 & 77,8 \\
$3,5-3,9$ & 8 & 14,8 & 14,8 & 92,6 \\
$4,0-5,0$ & 4 & 7,4 & 7,4 & 100,0 \\
Total & 54 & 100,0 & 100,0 & \\
& & & & \\
\hline
\end{tabular}

En el segundo parcial, se observa una leve mejoría en el desempeño del grupo experimental, el 60\% logró notas aprobatorias, disminuyendo de $44 \%$ a $40 \%$ la mortalidad académica; se destaca el aumento en el rango de 3,5 a 3,9, relacional, el cual pasó de $6 \%$ del primer parcial a $24 \%$ en el segundo. En el control, aumentó la mortalidad académica del 44,4\% del primer parcial al 55,6\% del segundo. Asimismo, disminuyó el porcentaje en el rango de 3,0 a 3,5, multiestructural, del $31,5 \%$ del primer parcial al $22,2 \%$ del segundo.

\section{Resultados del tercer parcial}

Tabla 17: Resultados del tercer parcial grupo experimental.

Rangos de desempeño tercer parcial

\begin{tabular}{|c|r|r|r|r|}
\hline & Frecuencia & Porcentaje & $\begin{array}{c}\text { Porcentaje } \\
\text { Válido }\end{array}$ & $\begin{array}{r}\text { Porcentaje } \\
\text { Acumulado }\end{array}$ \\
\hline Válidos $1,6-2,9$ & 7 & 14,0 & 14,0 & 14,0 \\
$3,0-3,4$ & 13 & 26,0 & 26,0 & 40,0 \\
$3,5-3,9$ & 19 & 38,0 & 38,0 & 78,0 \\
$4,0-5,0$ & 11 & 22,0 & 22,0 & 100,0 \\
Total & 50 & 100,0 & 100,0 & \\
& & & & \\
\hline
\end{tabular}

Tabla 18: Resultados del tercer parcial grupo control. Rangos de desempeño tercer parcial

\begin{tabular}{|c|r|r|r|r|}
\hline & Frecuencia & Porcentaje & $\begin{array}{c}\text { Porcentaje } \\
\text { Válido }\end{array}$ & $\begin{array}{c}\text { Porcentaje } \\
\text { Acumulado }\end{array}$ \\
\hline Válidos $1,6-2,9$ & 20 & 37,0 & 37,0 & 37,0 \\
$3,0-3,4$ & 20 & 37,0 & 37,0 & 74,1 \\
$3,5-3,9$ & 12 & 22,2 & 22,2 & 96,3 \\
$4,0-5,0$ & 2 & 3,7 & 3,7 & 100,0 \\
Total & 54 & 100,0 & 100,0 & \\
\hline
\end{tabular}

Se observa avance del experimental en estudiantes que lograron notas aprobatorias, pasando del 60\% del segundo parcial al $86 \%$ en el tercero. Se destaca el aumento en los niveles relacional y abstracto ampliado, pasando del $30 \%$ del segundo al $60 \%$ en el tercer parcial. La disminución en el multiestructural se refleja en aumento del relacional y del abstracto ampliado, que pasaron del $24 \%$ al $38 \%$ y del $6 \%$ al $22 \%$, respectivamente. En el grupo control hay aumento significativo en el multiestructural, que pasó del 31,5\% del primer parcial al 37,03\% del tercero, en concordancia con disminución en uniestructural, que pasó del $55,5 \%$ en el segundo al $37,0 \%$ del tercero.

\section{Resultados nota definitiva}

Tabla 19: Resultados examen definitivo grupo experimental.

Rangos de desempeño Nota definitiva

\begin{tabular}{|c|r|r|r|r|}
\hline & Frecuencia & Porcentaje & $\begin{array}{c}\text { Porcentaje } \\
\text { Válido }\end{array}$ & $\begin{array}{c}\text { Porcentaje } \\
\text { Acumulado }\end{array}$ \\
\hline Válidos $1,6-2,9$ & 8 & 16,0 & 16,0 & 16,0 \\
$3,0-3,4$ & 29 & 58,0 & 58,0 & 74,0 \\
$3,5-3,9$ & 6 & 12,0 & 12,0 & 86,0 \\
$4,0-5,0$ & 7 & 14,0 & 14,0 & 100,0 \\
Total & 50 & 100,0 & 100,0 & \\
\hline
\end{tabular}


En el experimental, la tendencia fue de aumento en los niveles relacional y abstracto ampliado, en concordancia con disminución en el uniestructural. El porcentaje que avanzó del uniestructural al multiestructural es significativo, evidenciando la importancia del proceso de intervención. La mortalidad final del grupo disminuyó al 14\%, aunque aún se considera alta, contrasta con el $44 \%$ del primer parcial.

Tabla 19: Resultados examen definitivo grupo control.

Rangos de desempeño Nota definitiva

\begin{tabular}{|c|r|r|r|r|}
\hline & Frecuencia & Porcentaje & $\begin{array}{c}\text { Porcentaje } \\
\text { Válido }\end{array}$ & $\begin{array}{c}\text { Porcentaje } \\
\text { Acumulado }\end{array}$ \\
\hline Válidos $1,6-2,9$ & 18 & 33,3 & 33,3 & 33,3 \\
$3,0-3,4$ & 31 & 57,4 & 57,4 & 90,7 \\
$3,5-3,9$ & 4 & 7,4 & 7,4 & 98,1 \\
$4,0-5,0$ & 1 & 1,9 & 1,9 & 100,0 \\
Total & 54 & 100,0 & 100,0 & \\
\hline
\end{tabular}

En este grupo no se evidencian cambios en el abstracto ampliado, en el cual se observan $7,4 \%$ para el primer y segundo parcial y una disminución en el tercero, al caer al 3,7\%. En el relacional, el comportamiento es inconstante, ya que disminuyó del $16,7 \%$ en el primero al $14,8 \%$ en el segundo, para aumentar de nuevo al 22,2\% en el tercero. Similarmente en el multiestructural, del $31,5 \%$ del primero, bajó al 22,2\% en el segundo, aumentando al 37,0\% en el tercero, ubicando aquí el mayor número de estudiantes, 57,4\%. En el uniestructural, el 33,3\% representa la mortalidad académica más alta de los dos grupos, evidenciando que los procesos de enseñanza tradicionales deben ser intervenidos para mejorar esos indicadores y la calidad de los aprendizajes.

\section{Análisis inferencial de los desempeños}

El resumen del comportamiento en los niveles de desempeño de los grupos durante el desarrollo del curso se muestra a continuación:

Tabla 21: Comportamiento en los niveles de desempeño grupo experimental.

\begin{tabular}{|c|c|c|c|c|c|c|c|c|c|}
\hline \multirow[b]{2}{*}{$\begin{array}{l}\text { Niveles de } \\
\text { comprensión }\end{array}$} & \multirow[b]{2}{*}{ Rango de notas } & \multicolumn{2}{|c|}{ Primer parcial } & \multicolumn{2}{|c|}{ Segundo parcial } & \multicolumn{2}{|c|}{ Tercer parcial } & \multicolumn{2}{|c|}{ Definitiva } \\
\hline & & $\begin{array}{l}\text { Número de } \\
\text { estudiantes }\end{array}$ & $\%$ & $\begin{array}{l}\text { Número de } \\
\text { estudiantes }\end{array}$ & $\%$ & $\begin{array}{l}\text { Número de } \\
\text { estudiantes }\end{array}$ & $\%$ & $\begin{array}{l}\text { Número de } \\
\text { estudiantes }\end{array}$ & $\%$ \\
\hline Preestructural & $0,0-1,5$ & & & & & & & & \\
\hline Uniestructural & $1,6-2,9$ & 22 & 44,0 & 20 & 40,0 & 7 & 14,0 & 8 & 16,0 \\
\hline Multiestructural & $3,0-3,4$ & 22 & 44,0 & 15 & 30,0 & 13 & 26,0 & 29 & 58,0 \\
\hline Relacional & $3,5-3,9$ & 3 & 6,0 & 12 & 24,0 & 19 & 38,0 & 6 & 12,0 \\
\hline Abstracto ampliado & $4,0-5,0$ & 3 & 6,0 & 3 & 6,0 & 11 & 22,0 & 7 & 14,0 \\
\hline & Total & 50 & 100 & 50 & 100 & 50 & 100,0 & 50 & 100 \\
\hline
\end{tabular}

Tabla 22: Comportamiento en los niveles de desempeño grupo control.

\begin{tabular}{|c|c|c|c|c|c|c|c|c|c|}
\hline \multirow[b]{2}{*}{$\begin{array}{l}\text { Niveles de } \\
\text { comprensión }\end{array}$} & \multirow[b]{2}{*}{ Rango de notas } & \multicolumn{2}{|c|}{ Primer parcial } & \multicolumn{2}{|c|}{ Segundo parcial } & \multicolumn{2}{|c|}{ Tercer parcial } & \multicolumn{2}{|c|}{ Definitiva } \\
\hline & & $\begin{array}{l}\text { Número de } \\
\text { estudiantes }\end{array}$ & $\%$ & $\begin{array}{l}\text { Número de } \\
\text { estudiantes }\end{array}$ & $\%$ & $\begin{array}{l}\text { Número de } \\
\text { estudiantes }\end{array}$ & $\%$ & $\begin{array}{l}\text { Número de } \\
\text { estudiantes }\end{array}$ & $\%$ \\
\hline Preestructural & $0,0-1,5$ & & & 1 & 1,9 & & & & \\
\hline Uniestructural & $1,6-2,9$ & 24 & 44,4 & 29 & 53,7 & 20 & 37,0 & 18 & 33,3 \\
\hline Multiestructural & $3,0-3,4$ & 17 & 31,5 & 12 & 22,2 & 20 & 37,0 & 31 & 57,4 \\
\hline Relacional & $3,5-3,9$ & 9 & 16,7 & 8 & 14,8 & 12 & 22,0 & 4 & 7,4 \\
\hline Abstracto ampliado & $4,0-5,0$ & 4 & 7,4 & 4 & 7,4 & 2 & 3,7 & 1 & $1,9+$ \\
\hline & Total & 54 & 100 & 54 & 100,0 & 54 & 100,0 & 54 & 100 \\
\hline
\end{tabular}


Los resultados se confirman mediante un análisis estadístico inferencial sobre las notas obtenidas por ambos grupos, durante los tres parciales y el examen definitivo. Se usó la prueba T para la diferencia de promedios, con Ho: $\mu_{1=} \mu_{2} \mathrm{Vs} \mathrm{H}_{1}: \mu_{1 \neq} \mu_{2}$ Se realizó mediante el software SPSS 10.0. El supuesto de igualdad de varianzas fue validado por medio de la prueba $\mathrm{F}$, hallando igualdad estadística de varianzas poblacionales. Para la prueba de hipótesis se estableció que Ho es o no rechazada con un valor especificado a o nivel de significancia. Se tomaron los valores de $1 \%$ y $5 \%$, los cuales son comparados con un p-valor o nivel de significancia más pequeño, que conduce al rechazo de la hipótesis Ho. Es decir, p-valores menores que un 0,05 conducen al rechazo de Ho, arrojando que estadísticamente hay diferencia significativa $\left(^{*}\right)$ entre los promedios y con $\mathrm{p}$-valores menores que 0,01 , se concluye que estadísticamente hay diferencia altamente significativa $\left(^{* *}\right)$. Los promedios en los tres parciales $y$ definitiva fueron:

Tabla 23: Promedios de cada parcial y definitiva correspondiente a cada grupo.

\begin{tabular}{|l|c|c|c|c|}
\hline Grupos & $\begin{array}{c}\text { Primer } \\
\text { parcial }\end{array}$ & $\begin{array}{c}\text { Segundo } \\
\text { parcial }\end{array}$ & $\begin{array}{c}\text { Tercer } \\
\text { parcial }\end{array}$ & Definitiva \\
\hline Control & 3,06 & 2,96 & 3,05 & 3,02 \\
\hline Experimental & 2,97 & 3,13 & 3,53 & 3,22 \\
\hline
\end{tabular}

Los resultados de la prueba de medias T muestran que en el grupo control no se observa diferencia significativa entre cada par de promedios de parciales $(p<0,05)$, mientras que para el experimental sólo los promedios del primer y segundo parcial no presentan diferencia significativa. Aunque no se observa desde el inicio el efecto del tratamiento sobre la variable dependiente (desempeño académico) en el grupo experimental, éste empieza a evidenciarse en el segundo y tercer parcial.

Tabla 24: P-valores de la prueba $\mathrm{T}$ para la diferencia de promedios de las notas parciales.

\begin{tabular}{|c|c|c|}
\hline & Grupo control & $\begin{array}{c}\text { Grupo } \\
\text { experimental }\end{array}$ \\
\hline $\begin{array}{c}\text { Primer parcial vs } \\
\text { segundo parcial }\end{array}$ & $0,371 \mathrm{~ns}$ & $0,104 \mathrm{~ns}$ \\
\hline $\begin{array}{c}\text { Segundo parcial vs } \\
\text { tercer parcial }\end{array}$ & $0,491 \mathrm{~ns}$ & 0 ** \\
\hline $\begin{array}{c}\text { Primer parcial vs } \\
\text { tercer parcia }\end{array}$ & $0,897 \mathrm{~ns}$ & 0 ** \\
\hline
\end{tabular}

La prueba de medias T, para la diferencia de promedios entre los respectivos parciales de los grupos, mostró una diferencia altamente significativa entre los promedios del tercer parcial y las definitivas $(\mathrm{p}<0,05)$, mostrando un efecto del tratamiento en la mejoría del promedio de notas en el grupo experimental a partir de los terceros parciales.

Tabla 25: P-valores de la prueba $\mathrm{T}$ para la diferencia de promedios de parciales y definitiva.

\begin{tabular}{|l|l|l|}
\hline \multicolumn{3}{|c|}{ Grupo control vs grupo experimental } \\
\hline \multicolumn{1}{|c|}{ Grupo control } & \multicolumn{1}{|c|}{ Grupo experimental } & \multicolumn{1}{|c|}{ P-valor } \\
\hline Primer parcial & Primer parcial & 0,397 \\
Segundo parcial & Segundo parcial & 0,109 \\
Tercer parcial & Tercer parcial & $0 * *$ \\
Definitiva & Definitiva & $0,008 * *$ \\
\hline
\end{tabular}

Se llevó a cabo un análisis de intervalo de confianza al 95\% para aquellos casos en los que estadísticamente se presentó diferencia de promedios, con el fin de determinar cuál promedio de parcial fue superior entre los grupos.

Tabla 26: Intervalos de confianza para la diferencia de promedios.

\begin{tabular}{|l|c|c|}
\hline \multicolumn{1}{|c|}{ Promedios de nota parcial } & $\begin{array}{c}\text { Limite } \\
\text { inferior }\end{array}$ & Limite superior \\
\hline $\begin{array}{l}\text { Primer a tercer parcial grupo } \\
\text { experimental }\end{array}$ & $-0,753$ & $-0,355$ \\
\hline $\begin{array}{l}\text { Segundo a tercer parcial grupo } \\
\text { experimental }\end{array}$ & $-0,597$ & $-0,195$ \\
\hline Tercer parcial entre grupos & $-0,681$ & $-0,281$ \\
\hline Definitivas entre grupos & $-0,346$ & $-0,053$ \\
\hline
\end{tabular}


En todos los casos se aprecian intervalos de confianza negativos, es decir, la diferencia entre los promedios de notas parciales es estadísticamente negativa, por lo que se infiere que en el experimental el promedio del tercer parcial es mayor al del segundo y al del tercero. Los promedios del tercer parcial y nota definitiva en el grupo experimental son mayores al compararlos con los del grupo control.

\section{Conclusiones}

- Existe un efecto positivo de la aplicación de estrategias mediadas con TIC sobre el desempeño académico del grupo experimental, evidenciado en el aumento paulatino de las notas y de los niveles de comprensión, en la medida en que evoluciona su proceso de aplicación.

- Aunque los dos grupos inicialmente (pronóstico) presentaron condiciones similares en cuanto a desempeño académico, el experimental, al utilizar estrategias de enfoque profundo (mediadas con TIC) mejoró significativamente su desempeño (producto), en contraste con las estrategias de enfoque superficial utilizadas por el grupo control e identificadas en el proceso, evidenciando que los fracasos están asociados no solamente a las condiciones internas del estudiante, sino a un conjunto de condiciones externas y a las estrategias utilizadas en el proceso.

\section{Bibliografía}

Biggs, J.(1996). Enhancing teaching through constructive alignment, higher Education. Sydney: Prentice Hall of Australia.

Biggs, J. (2005). Calidad del aprendizaje universitario. Madrid: Nancea de Ediciones.

Biggs, J., Collins, K. F. (1982). Evaluating the quality of learning: the Solo Taxonomy. Nueva York: Academic Press.

Carrascal, N. (2006). Estilos, estrategias de aprendizaje, contextos de enseñanza y su influencia en la calidad de los resultados académicos en estudiantes de educación superior. Memoria para la obtención del Diploma de Estudios Avanzados. España: Universidad de Granada.
- La aplicación de la propuesta basada en perspectivas socio-constructivistas, enfatizando la importancia de la actividad del estudiante y de su interacción con el contexto, constituyéndose en un proceso cognitivo, mediado y activo, significativo y completo, generó en el grupo experimental cambios significativos:

- En cuanto a niveles de comprensión, el grupo evolucionó hacia niveles superiores, pasando del $50 \%$ en el nivel uniestructural, $33 \%$ en multiestructural, $13 \%$ en el relacional y $3 \%$ en el abstracto ampliado, presentados en el inicio del curso, hasta un $14 \%$ en el nivel uniestructural, $26 \%$ en el multiestructural, $38 \%$ en el relacional y $22 \%$ en el abstracto ampliado al final del curso.

- La mortalidad académica del grupo (16\%) disminuyó significativamente respecto a la del grupo control, que se situó en el 33\%.

- Los resultados obtenidos en el grupo experimental demuestran la eficacia de la estrategia para el desarrollo del aprendizaje por competencias y centrado en el estudiante. En concreto, los estudiantes del grupo experimental, al final del curso, mejoraron significativamente sus conocimientos, sus estrategias de aprendizaje; disminuyeron el uso de un enfoque superficial y mejoraron en la calidad de las tareas evaluadas con la Taxonomía Solo. 그

Coll, C. (1988). Conocimiento psicológico y práctica educativa: introducción a la relación entre psicología y educación. Madrid: Barcanova.

Coll, C. (1990). Psicología y educación: Aproximación a los objetivos y contenidos de la psicología de la educación. En C. Coll, J. Palacios y A. Marchesi (comp.), Desarrollo psicológico y educación. Madrid: Alianza.

Conaces. (2004). Memorias del encuentro de miembros de las salas de la Comisión Intersectorial de Aseguramiento de la Calidad de la Educación Superior. Bogotá, 8 de octubre de 2004.

González, A. et al. (1996). Las nuevas tecnologías en la educación. En Salinas et al., Redes de comunicación, 
redes de aprendizaje. EDUTEC'95, 409-422. Obtenido el 22 de abril, 2007 de http://www.uib.es/depart/ gte/grurehidi.html

Hernández, P. (2007). Tendencias de Web 2.0 aplicadas a la educación en línea. No Solo Usabilidad, 6. Obtenido el 12 de abril de 2008 de http://www.nosolousabilidad.com/articulos/web20.htm

Hernández Pina, F. (2002). Docencia e investigación en educación superior. Revista de Investigación Educativa, 20(2), 270-301.

Hernández Pina, F. et al. (2005). Aprendizaje, competencias y rendimiento en Educación Superior. Madrid: La Muralla.
Hernández, R. et al. (1999). Metodología de investigación. México: McGraw Hill.

Marton, F y Saljo, R. (1976). On qualitative differences in learning-I: Out-come and process. British Journal of educational Psychology, 4-11.

Romizowsky, A. J. (1981). Designing instructional systems. Londres: Kogan Page.

Unesco. (1998). Declaración mundial sobre la educación superior en el siglo XXI: Visión y acción y marco de acción prioritaria para el cambio y el desarrollo de la educación superior. París, 5 al 9 de octubre. 